\title{
Oilfield Produced Water Reuse and Reinjection with Membrane
}

\author{
Utjok W.R. Siagian ${ }^{1}$, Setyo Widodo ${ }^{2}$, Khoiruddin $^{3}$, Anita K. Wardani ${ }^{3}$, and I Gede Wenten ${ }^{3, *}$ \\ ${ }^{1}$ E Department of Petroleum Engineering, Institut Teknologi Bandung, Jl. Ganesha 10, Bandung 40132, Indonesia \\ ${ }^{2}$ PPPTMGB LEMIGAS, Balitbang KESDM, Jl. Ciledug Raya, Kav. 109, Cipulir, Kebayoran Lama, Jakarta 12230, Indonesia \\ ${ }^{3}$ Department of Chemical Engineering, Institut Teknologi Bandung, J1. Ganesha 10, Bandung 40132, Indonesia
}

\begin{abstract}
Produced water has become a global environmental issue due to its huge volume and toxicity that may pose detrimental effects on receiving environment. Several approaches have been proposed to provide a strategy for produced water handling such as reinjection, reuse, or discharge. With various advantages, membrane technology has been increasingly used in produced water treatment replacing the conventional technologies. However, fouling is a major drawback of membrane processes in this application which needs to be controlled. This paper gives an overview and comparison of different produced water management. Special attention is given to produced water treatment for reuse purpose. Furthermore, the use of membrane processes in produced water reuse including performance, challenges, and future outlook are discussed.
\end{abstract}

\section{Introduction}

Hydrocarbon exploration and exploitation activities pose a serious environmental problem due to the production of produced water stream, which is also the largest portion of the waste streams $[1,2]$. Produced water can be defined as trapped water under a geological structure that comes along to the surface as oil and gas are being drilled out [3]. Components of produced water vary from one location to another depending on geological formation, geological condition and types of hydrocarbon produced [4]. Most of the constituents include dispersed oil, organic and inorganic contaminants, treatment chemical (from oil production process such as emulsion breaker, corrosion and scaling inhibitor, coagulant, etc.), produced solids, bacteria, metal and naturally occurring radioactive material (NROM) $[1,3]$. As the major constituent, oily substances and grease [3] with alkanes, alkenes, alkynes aromatic and complex hydrocarbon compounds have become the primary concern in produced water treatment [5]. On the other hand, a high salt concentration of produced water also becomes a problem when the onshore operation takes place [3].

Produced water is usually managed by several ways such as production avoidance, produced water injection, reuse and consumption of treated produced water for beneficial usages [6]. According to the reported studies on produced water treatment, discharge is the most common practice of produced water treatment (Fig. 1). The hazardous constituents in produce water should be removed before being discharged directly [2]. Studies on searching a better method for produced water treatment is increasing due to a more stringent regulation of produced water discharge. Meanwhile, other efforts are conducted to find a cost-effective method for produced water reuse since it is considered as an unconventional water source that may be used for beneficial usages such as industrial water, agriculture, potable water, and reinjection into a nearby formation well for enhancing oil recovery. In the reuse water purpose, it is important to significantly reduce the contaminants by choosing a proper treatment to meet the requirement of water quality. In addition, there are two general objectives in produced water treatment: removal of undesired constituents (excessive hardness, dissolved solids, microorganisms, light hydrocarbon, etc.) and effluent adjustment when it is going to be re-used such as for irrigation [6].

Four methods of produce water treatment have been proposed, i.e. physical, chemical, biological and membrane-based treatment [1]. With the ability of producing a high quality product along with relatively lower cost, lower energy and chemical consumption, smaller footprint, more intensive process, easy to scaleup, and flexible to operate [7-13], membrane is considered as an attractive technology in various processes including in water and wastewater treatment [14-19]. In produced water treatment, the number of publications on membrane applications in the last two decades is increasing which indicates the gaining attention on this technology (Fig. 1). This increasing attention is due to the ability of membrane in producing a high quality effluent that meet the required standard either for discharge and reuse purposes.

\footnotetext{
* Corresponding author: igw@che.itb.ac.id
} 


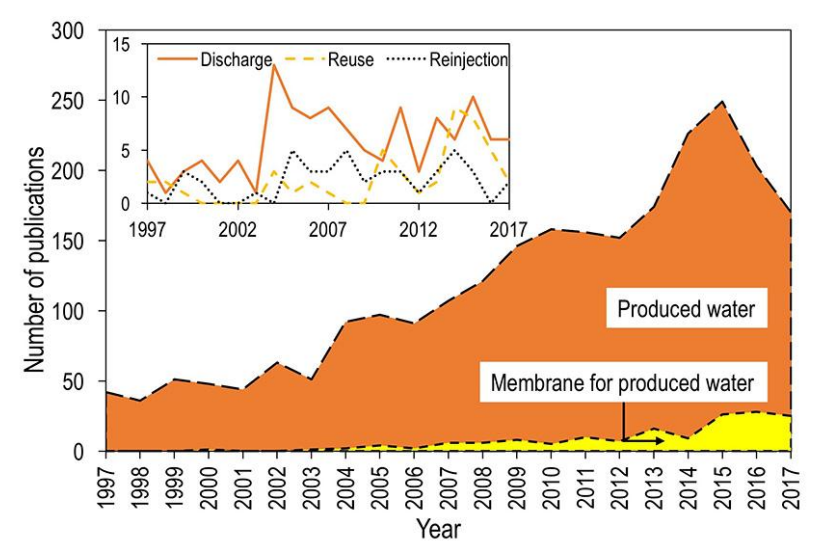

Fig. 1. Publications of produced water in the last 20 years indexed by Scopus (query: TITLE ("terms"))

Several papers reviewing the performance and applications of membrane technology in produced water treatment can be found [20-24]. In this paper, membrane technology for produced water treatment is reviewed focusing on reuse purpose. Current progress and challenges of membrane technology in this application are discussed.

\section{Produced water treatment purposes}

\subsection{Produced water characteristics}

Typical characteristics of produced water treatment reported in some studies are tabulated in Table 1. Produced water contains a mixture of inorganic and organic substances, solids, a small fraction of dissolved hydrocarbons, and gasses together with chemicals used for enhanced oil recovery $[25,26]$. These contaminants can be classified as follows: high total dissolved solids, suspended solid, dispersed oil, dissolved and volatile organic compounds, heavy metals, radionuclides, dissolved gases, and bacteria [27]. Both produced water volume and characteristics highly depends on formation well or reservoir including its geological characteristics, lifetime, and the hydrocarbon produced [28,29]. The volume of produced water increases along with increasing reservoir lifetime. The characteristics and the large volume are challenging for produced water handling. Inappropriate treatment may result in negative effect when it is discharged to a receiving environment. On the other hand, the large volume of produced water may be considered as a potential unconventional water source that can be used for various beneficial purposes [30]. For this purpose, a large fraction of components should be removed to meet the specifications of desired water quality. Standard quality of produced water discharge, reuse, and reinjection have been summarized by $\mathrm{Li}$ and Lee [31] as follow:

- Offshore disposal: suspended solid $<10 \mathrm{mg} / \mathrm{L}$; oil $<5$ $\mathrm{mg} / \mathrm{L}$;

- Reinjection: Suspended solid $<1 \mathrm{mg} / \mathrm{L}$; particle size of solid $<1 \mu \mathrm{m}$; Oil content $<5 \mathrm{mg} / \mathrm{L}$;

- Irrigation: Oil and grease $<35 \mathrm{mg} / \mathrm{L}$; TDS $=500$ -
$2000 \mathrm{mg} / \mathrm{l}$

- Cooling tower: TDS $<2700 \mathrm{mg} / \mathrm{L}$;

- Chemical process: TDS $<1000 \mathrm{mg} / \mathrm{L}$;

\subsection{Produced water discharge}

Generally, produced waters are discharged or reused. Produced water is usually discharged into the sea (for offshore), local river (for onshore), and estuarine or coastal water (for terminal or oil refinery) [26]. Due to a more stringent regulation of produced water discharge, the effective treatment technologies are required. Before disposal, produced water undergoes several steps of treatment including (i) removal of suspended solids and dispersed hydrocarbon and (ii) removal of oil and total carbon content, so the oil contents below $10 \mathrm{mg} / \mathrm{L}$ [32]. The other standards are stated by OSPAR (Convention for the Protection of the Marine Environment of the North-East Atlantic) and EPA (USA Environment Protection Agency). The standards are as follow [33]: (1) in OSPAR: Dispersed aliphatic oil $=30 \mathrm{mg} / \mathrm{L}$; oil and grease $=n / a$ and (2) in EPA: Dispersed aliphatic oil $=$ $\mathrm{n} / \mathrm{a}$; oil and grease $=42 \mathrm{mg} / \mathrm{L}$. This standard is focused on offshore discharge practice. It should be noted that dispersed hydrocarbon is not the only one component which becomes the environmental concern [34]. For onshore, many more parameters should be taken into account [35]. The dissolved solids, COD, BOD, heavy metals and other toxic contaminants should also be removed to avoid pollution in receiving water bodies [35].

\subsection{Produced water reuse and reinjection}

Since the source of fresh water starts to diminish, the large volume of produced water has been considered as a potential alternative which could be reclaimed for industrial purposes and directly reduces freshwater withdrawals [36]. This will also reduce the discharge of waste effluent to environment thus minimizing the environmental risk. Produced water reuse provides more beneficial economic and environmental impacts rather than discharge. Most of the produced water is reused for water injection. The purpose of reinjection of produced water into formation wells is to enhance oil recovery [37]. Reinjection is also aimed for disposal purpose. The attractive goals of both saving fresh water usage along with reducing waste disposal or near-zero discharge made reinjection as an example of smart water source management which turns waste into resource [38]. It is also viewed as a more cost-effective alternative rather than discharge and conventional waterflooding $[39,40]$. According to Robinson [41], the advantages of produced water reinjections include increasing hydrocarbon production to around $40 \%$ and removing an extensive surface water treatment system. In addition, using produced water as injection also offers some advantages, such as available in oil production field, compatible, near zero bacteria, and solve disposal problem [41]. 
Table 1. Typical characteristics of produced water reported in several studies.

\begin{tabular}{|c|c|c|c|c|c|c|c|c|}
\hline TDS $(\mathrm{mg} / \mathrm{L})$ & $\begin{array}{c}\text { Conductivity } \\
(\mu \mathrm{S} / \mathrm{cm})\end{array}$ & $\begin{array}{c}\mathrm{COD} \\
(\mathrm{mg} / \mathrm{L})\end{array}$ & $\begin{array}{c}\mathrm{BOD} \\
(\mathrm{mg} / \mathrm{L})\end{array}$ & $\begin{array}{c}\text { TOC } \\
(\mathrm{mg} / \mathrm{L}\end{array}$ & $\begin{array}{c}\text { Oil and } \\
\text { grease }(\mathrm{mg} / \mathrm{L})\end{array}$ & $\begin{array}{c}\text { TSS } \\
(\mathrm{mg} / \mathrm{L})\end{array}$ & $\mathrm{pH}$ & Ref. \\
\hline- & 47,600 & 588 & - & - & - & - & 7.1 & {$[42]$} \\
\hline 80,470 & - & 1622 & 695 & 386 & 220 & 167 & 6.8 & {$[43]$} \\
\hline- & $20,000-80,000$ & $1000-9000$ & - & - & $200-5000^{\mathrm{a}}$ & - & $6.0-8.0$ & {$[32]$} \\
\hline- & - & $380-490$ & - & - & $50-200$ & $30-160$ & $8.0-9.0$ & {$[44]$} \\
\hline 6000 & - & - & - & 120 & 50 & - & 7.0 & {$[45]$} \\
\hline $5500-7000$ & - & - & - & - & $0-60^{\mathrm{b}}$ & - & $6.0-8.0$ & {$[46]$} \\
\hline $98,800^{\mathrm{d}}$ & 100,000 & $156^{\mathrm{c}}$ & - & 113 & - & 60 & 6.6 & {$[47]$} \\
\hline $3100-5800$ & - & $450-1280$ & - & - & $12.5-84.4^{\mathrm{e}}$ & $7.9-89.2$ & $7.2-8.1$ & {$[48]$} \\
\hline 6000 & - & - & - & 120 & 20 & - & 7.0 & {$[49]$} \\
\hline- & $20,000-80,000$ & - & - & $200-2000$ & $32-180^{\mathrm{a}}$ & - & $7.0-7.8$ & {$[28]$} \\
\hline 28,980 & 46,000 & 1440 & - & 403 & 14 & - & - & {$[36]$} \\
\hline 96,472 & - & - & - & $268.2^{\mathrm{f}}$ & $120.4^{\mathrm{g}}$ & 20.2 & 8.7 & {$[50]$} \\
\hline 12,900 & - & 3000 & - & - & 28 & - & 6.77 & {$[51]$} \\
\hline $10,000-17,500$ & - & - & - & $48-164$ & $3-220^{\mathrm{e}}$ & $6-110$ & 8.3 & {$[52]$} \\
\hline
\end{tabular}

${ }^{\mathrm{a}}$ Dispersed oil; ${ }^{\mathrm{b}}$ Free oil; ${ }^{\mathrm{c}} 3 *$ DOC (equation from dowac.custhelp.com); ${ }^{\mathrm{d}}$ Salt concentration; ${ }^{\mathrm{e}}$ Oil; ${ }^{\mathrm{f}}$ Dissolved organic including hydrocarbon and soluble oil; ${ }^{\mathrm{g}}$ Free oil droplets; - Data not available;

Nevertheless, the treated produced water should be compatible with the reservoir. For instance, the presence of oil and suspended solid smay cause reservoir interface plugging [39]. Therefore, the component which causes plugging should be removed. Specification of injection water may vary depending on formation characteristics. For low-permeability formations, the injection water should have $<1 \mathrm{mg} / \mathrm{L}$ of total hydrocarbons and $<10$ $\mathrm{mg} / \mathrm{L}$ of suspended solids, and $<1 \mu \mathrm{m}$ of particle size [31].

Moreover, for designing an injection water, some the following parameters should be taken into account: (i) size distribution of oil droplet, (ii) oil content, (iii) size distribution of suspended solids, (iv) total suspended components, (v) dissolved gas, (vi) bacteria, and (v) scaling potential [34].

The large volume of produced water is also viewed as potential source of water for various usages such as irrigation, potable water, and industrial process water. However, the complex mixture of pollutants presents the challenges for achieving treatment steps that both cost effective and high efficiency [27,53]. For example, the high-quality standard and stringent regulation for drinking water applications lead to the complex treatment steps which result in a high treatment cost [54]. Therefore, numerous efforts were made to develop more economical and advanced treatment technologies to meet various end-use requirements.

\section{Membrane in produced water reuse and reinjection}

The use of membrane technology in produced water treatment is increasing since the conventional technologies are no longer capable to meet the more stringent regulation in produced water treatment. Membrane process may be combined with conventional processes. Membrane-based integrated processes are also reported by several studies to obtain a high quality reclaimed water.

\footnotetext{
${ }^{*}$ Corresponding author: igw@,che.itb.ac.id
}

Generally, produced water treatment includes several steps namely pre-treatment, main treatment, polishing treatment, and tertiary treatment [27]. Coarse particles, oil, and gas are removed in pre-treatment step. The main step is usually used to remove small hydrocarbon droplets and small particles resulting in dispersed hydrocarbon content to below $40 \mathrm{mg} / \mathrm{L}$. Further, produced water may undergo polishing and additional treatment depending on the final effluent quality needed.

Microfiltration (MF) and ultrafiltration (UF) membranes are used in several studies to reduce the oil content to an acceptable level. Performance of MF and UF membrane in produced water treatments are shown in Table 2 and Fig. 2. As indicated by Fig. 2, MF and UF can achieve $>99 \%$ oil removal from produced water. Furthermore, UF membrane with pore size of $0.05 \mu \mathrm{m}$ can remove $99 \%$ of oil with $39 \%$ of TOC [28].

Integrated $\mathrm{MF} / \mathrm{UF} / \mathrm{NF}$ or $\mathrm{RO}$ process is used when a high quality reclaimed water is required. Typical examples of the integrated process are summarized in Table 3. Ebrahimi et al [28] reported a study on produced water treatment using integrated MF, UF, and nanofiltration (NF). This integrated process successfully removed oil up to $99 \%$ with acceptable TOC removal. This study also suggested MF as a first efficient step for oil removal. Khedr [50] investigated the performance of integrated chlorination, coagulation, and NF membrane for produced water treatment. Coagulation can produce effluent with a low concentration of suspended solids, organic, and oil. Meanwhile, the introduction of NF as polishing step help to remove metal cations and to further remove suspended solids, bacteria, and organics. This polishing step is expected to inhibit scale and biofilm formation during injection. 


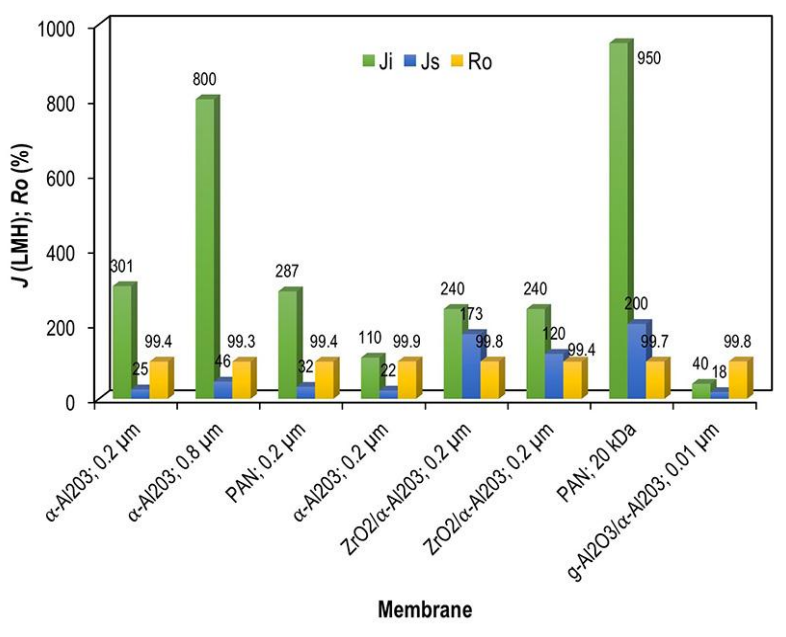

Fig. 2. Initial and stabilized flux $(J i, J S)$ and oil removal $(R o)$ of several MF and UF membrane during produced water and oily waste water treatment (data from refs. [55-58])

Integrated UF/NF/RO system was studied by Hayatbakhsh et al [59] to treat produced water from steam-assisted gravity drainage (SAGD). SAGD is generally used to ehance oil recovery for bitumen extraction from oil sands. The treatment purpose is to reclaim produced water for a high quality produced water wherein salt, silica, and dissolved organic matter are the concern. They found the UF membrane was able to remove up to $30 \%$ of salt and silica and $50 \%$ of dissolved organic matter. Tight NF membrane could remove $>86 \%$ of salts, silica, and dissolved organic matter.

Besides pressure driven membrane processes (MF, UF, NF, and RO), membrane bioreactor (MBR), a hybrid membrane/bioreactor process, is also a potential membrane process for produced water treatment. Submerged MBR equipped with PVDF membrane was used by Sun et al [60] to produce water injection from produced water. The effluent of $\mathrm{MBR}$ contained $<1$ $\mathrm{mg} / \mathrm{L}$ of crude oil, $<1 \mathrm{mg} / \mathrm{L}$ of $\mathrm{NH}_{4}{ }^{+}-\mathrm{N}$, and $1 \mathrm{NTU}$ of turbidity. This effluent was able to achieve the grade A1 criteria of Recommendation Standard and Analysis Method for the Clastic Rock Reservoir Injection Water Quality (SY/T 5329-94, China).

Table 2. Performance of MF and UF membrane in produced water treatment

\begin{tabular}{|c|c|c|c|c|}
\hline $\begin{array}{l}\text { Membrane; Pore size or } \\
\text { MWCO. }\end{array}$ & Operating conditions & Feed water & Results & Ref. \\
\hline $\begin{array}{c}\text { Ceramic membrane } \\
\left(93.32 \% \mathrm{SiO}_{2}\right)\end{array}$ & 1 bar & $\begin{array}{l}\text { Synthetic oil } / \text { water; } \mathrm{C}_{\text {oil }}= \\
200 \mathrm{mg} / \mathrm{L} .\end{array}$ & $\begin{array}{c}\text { Oil in permeate }=2 \mathrm{mg} / \mathrm{L}(99,8 \% \\
\text { rejection) }\end{array}$ & [65] \\
\hline $\begin{array}{c}\text { Mixed cellulose esters; } \\
0.1 \mu \mathrm{m} \\
\end{array}$ & $\begin{array}{c}0.5 \text { bar; } \mathrm{CFV}=0.43 \\
\mathrm{~m} / \mathrm{s} ;\end{array}$ & Produced water & $\begin{array}{c}\text { Removal: } \text { COD }=35 \% ; \text { TOC }=25 \% \\
\text { O\&G }=92 \% ; \text { Phenol }=35 \%\end{array}$ & [43] \\
\hline $\mathrm{Al}_{2} \mathrm{O}_{3} ; 0.2 \mu \mathrm{m}$ & 1 bar; $60^{\circ} \mathrm{C}$ & Produced water & Oil removal $=93 \%$ & [66] \\
\hline $\begin{array}{c}\mathrm{ZrO}{ }_{2}(\text { active layer }) \\
\mathrm{TiO}_{2} / \mathrm{Al}_{2} \mathrm{O}_{3} \\
\end{array}$ & $\begin{array}{c}1.5 \text { bar; } 45^{\circ} \mathrm{C} ; \mathrm{CFV}=3 \\
\mathrm{~m} / \mathrm{s} ; \text { Pilot scale; }\end{array}$ & Produced water & $\begin{array}{c}\text { Oil in product }<5 \mathrm{mg} / \mathrm{L} ; \mathrm{SS}=\text { not } \\
\text { detected; Perm. }=620-1200 \mathrm{LMH} / \mathrm{bar}\end{array}$ & [31] \\
\hline$\alpha$-alumina; $0.8 \mu \mathrm{m}$ & 0.7 bar; $40^{\circ} \mathrm{C} ; 0.91$ & $\begin{array}{l}\text { Synthetic produced water; } \\
\mathrm{C}_{\text {oil }}=250 \mathrm{mg} / \mathrm{L}\end{array}$ & Oil removal = 99.3\%; & [55] \\
\hline$\alpha$-alumina; $0.2 \mu \mathrm{m}$. & 0.7 bar; $40^{\circ} \mathrm{C} ; 0.24$ & $\begin{array}{l}\text { Synthetic produced water; } \\
\text { Coil }_{\text {oil }}=1000 \mathrm{mg} / \mathrm{L}\end{array}$ & Oil removal $=99.4 \%$ & [55] \\
\hline PAN; $0.1 \mu \mathrm{m}$. & 1.4 bar; $40^{\circ} \mathrm{C} ; 0.24$ & $\begin{array}{l}\text { Synthetic produced water; } \\
\mathrm{C}_{\mathrm{oil}}=250 \mathrm{mg} / \mathrm{L}\end{array}$ & Oil removal $=99.8 \%$ & [55] \\
\hline $\begin{array}{c}\text { Cysteic acid } \\
\text { functionalized } \alpha \text { - } \\
\text { alumina; } 0.22 \mu \mathrm{m} .\end{array}$ & $\begin{array}{l}0.55 \text { bar; Single-pass } \\
\text { closed loop batch } \\
\text { system. }\end{array}$ & $\begin{array}{l}\text { Produced water; TOC }= \\
43,550 \mathrm{mg} / \mathrm{L}\end{array}$ & TOC removal $=98.5 \%$ & [67] \\
\hline$\alpha$-alumina; $1 \mu \mathrm{m}$. & $\begin{array}{c}1-1.5 \text { bar; } C F V=3-5 \\
\mathrm{~m} / \mathrm{s} .\end{array}$ & $\begin{array}{l}\text { Synthetic oil } / \text { water mixture; } \\
\qquad \mathrm{C}_{\text {oil }}=5000 \mathrm{mg} / \mathrm{L}\end{array}$ & $\begin{array}{c}\text { Oil removal }=94.3 \% ;(<10 \mathrm{mg} / \mathrm{L} \text { in } \\
\text { permeate }) .\end{array}$ & [56] \\
\hline$\alpha$-alumina; $0.2 \mu \mathrm{m}$. & $\begin{array}{c}1-1.5 \text { bar; } C F V=3-5 \\
\text { m/s. }\end{array}$ & $\begin{array}{l}\text { Synthetic oil/water mixture; } \\
\quad \mathrm{C}_{\text {oil }}=5000 \mathrm{mg} / \mathrm{L}\end{array}$ & $\begin{array}{c}\text { Oil removal }=99.9 \%(<10 \mathrm{mg} / \mathrm{L} \text { in } \\
\text { permeate }) .\end{array}$ & {$[56]$} \\
\hline $\mathrm{ZrO}_{2} / \alpha$-alumina; $0.2 \mu \mathrm{m}$. & $\begin{array}{l}1-1.5 \text { bar; } C F V=3-5 \\
\text { m/s. }\end{array}$ & $\begin{array}{l}\text { Synthetic oil } / \text { water mixture; } \\
\mathrm{C}_{\text {oil }}=5000 \mathrm{mg} / \mathrm{L}\end{array}$ & $\begin{array}{c}\text { Oil removal }=99.8 \%(<10 \mathrm{mg} / \mathrm{L} \text { in } \\
\text { permeate }) .\end{array}$ & [56] \\
\hline $\mathrm{ZrO}_{2} / \alpha$-alumina; $0.2 \mu \mathrm{m}$. & $\begin{array}{c}1.1 \mathrm{bar} ; \mathrm{CFV}=2.56 \\
\mathrm{~m} / \mathrm{s} ; 25^{\circ} \mathrm{C} ; \text { Flocculation } \\
(3530 \mathrm{~S}, 40 \mathrm{mg} / \mathrm{L})\end{array}$ & $\begin{array}{l}\text { Oily wastewater produced } \\
\text { from refinery process; } \mathrm{C}_{\text {oil }} \\
=6000 \mathrm{mg} / \mathrm{L} ; \mathrm{COD}=3 \\
\mathrm{~g} / \mathrm{L} ; \text { Solid }=5 \% \text {-wt. }\end{array}$ & $\begin{array}{l}\text { Oil removal }=99.8 \%(8.762 \mathrm{mg} / \mathrm{L} \text { in } \\
\text { permeate }) ; \text { COD in permeate }=108 ;\end{array}$ & [57] \\
\hline $\begin{array}{c}\gamma \text {-alumina/ } \alpha \text {-alumina; } \\
0.01 \mu \mathrm{m}\end{array}$ & $\begin{array}{c}1-1.5 \text { bar; } C F V=3-5 \\
\text { m/s. }\end{array}$ & $\begin{array}{l}\text { Synthetic oil } / \text { water mixture; } \\
\quad \mathrm{C}_{\text {oil }}=5000 \mathrm{mg} / \mathrm{L} \text {; }\end{array}$ & $\begin{array}{c}\text { Oil removal }=99.8 \%(<10 \mathrm{mg} / \mathrm{L} \text { in } \\
\text { permeate }) .\end{array}$ & [56] \\
\hline $\mathrm{TiO}_{2} ; 0.05 \mu \mathrm{m}$ & 1 bar; $60^{\circ} \mathrm{C}$ & Produced water & $\begin{array}{c}\text { Oil removal }>99 \% \text {; TOC removal }= \\
13.6 \% ;\end{array}$ & [68] \\
\hline $\mathrm{Al}_{2} \mathrm{O}_{3} ; 40 \mathrm{~nm}$ & $\begin{array}{l}0.5 \mathrm{bar}, \mathrm{CFV}=2 \mathrm{~m} / \mathrm{s} \\
\text { Total recycle; }\end{array}$ & Produced water & Oil removal $>99.5 \%$; & [32] \\
\hline PAN; 20 kDa & $\begin{array}{l}\text { TMP: } 3 \text { bar; T: 40oC; } \\
\text { CFV: } 1 \mathrm{~m} / \mathrm{s}\end{array}$ & $\begin{array}{c}\text { Oily wastewater; O\&G = } \\
78 \mathrm{mg} / \mathrm{L} ; \mathrm{TSS}=60 \mathrm{mg} / \mathrm{L} \\
\text { TOC }=81 \mathrm{mg} / \mathrm{L}\end{array}$ & $\begin{array}{l}\text { Final effluent: } O \& G=0.2 \mathrm{mg} / \mathrm{L} ; \mathrm{TSS} \\
=\text { trace; } \mathrm{TOC}=19 \mathrm{mg} / \mathrm{L}\end{array}$ & [58] \\
\hline
\end{tabular}


Table 3. Produced water treatment schemes and purposes

\begin{tabular}{|c|c|c|c|c|}
\hline No. & System configuration & Purpose & Remarks & Ref. \\
\hline 1 & $\begin{array}{l}\text { A primary sedimentation }+ \text { oil/water separator } \\
+ \text { DAF system }+1 \mu \mathrm{m} \text { cartridge filter }+0.2 \\
\mu \mathrm{m} \text { ceramic } \mathrm{MF}+\text { Activated carbon }+\mathrm{RO}\end{array}$ & Discharge & $\begin{array}{l}\text { Feed: Devecatagi oil well produced water; } \\
\text { Product: } \mathrm{COD}=108 \mathrm{mg} / \mathrm{L} ; \mathrm{TDS}=1726 \mathrm{mg} / \mathrm{L} ; \\
\qquad \mathrm{pH}=7\end{array}$ & [42] \\
\hline 2 & $\mathrm{MF}+$ biological treatment; & Discharge & $\begin{array}{l}\text { Feed: Campos basin oilfield wastewater; Final } \\
\text { effluent: COD: } 230 \mathrm{mg} / \mathrm{L} \text {, TOC: } 55 \mathrm{mg} / \mathrm{L} \text {, } \\
\text { respectively. }\end{array}$ & [43] \\
\hline 3 & $\begin{array}{l}\text { Induced gas floatation + walnut shell filter }+ \\
\text { heat exchanger }+ \text { DOX stripper }+ \text { chemical } \\
\text { addition + flocculation + heat exchanger }+ \\
\text { multimedia filter + cartridge filter }+ \text { RO (two- } \\
\text { pass) + constructed wetlands; } 79,493 \mathrm{~m}^{3} / \mathrm{d} \text {. }\end{array}$ & $\begin{array}{l}\text { Surface } \\
\text { discharge } \\
\text { to local } \\
\text { ground } \\
\text { water }\end{array}$ & $\begin{array}{c}\text { Feed: Produced water from San Ardo water } \\
\text { reclamation facility; Final effluent: TDS }=180 \\
\mathrm{mg} / \mathrm{L} ; \mathrm{Na}=50 \mathrm{mg} / \mathrm{L} ; \mathrm{Cl}=11 \mathrm{mg} / \mathrm{L} ; \text { Sulfate: } \\
125 \mathrm{mg} / \mathrm{L} ; \text { Nitrit = non-detectable; Boron }=0.10 \\
\text { mg } / \mathrm{L} ;\end{array}$ & [46] \\
\hline 4 & UF + RO + MED; Pilot plant. & $\begin{array}{l}\text { Reuse for } \\
\text { irrigation }\end{array}$ & $\begin{array}{l}\text { Feed: coal seam gas (CSG) produced water. } \\
\text { Clean water recovery }=95 \% \text {; A final brine } \\
\text { containing mostly sodium bicarbonate up to of } \\
48 \mathrm{~g} / \mathrm{L} . \text { RO recovery }=76 \% \text {; MED recovery= } \\
80 \% \text {. RO permeate and MED distillate were of } \\
\text { high quality and could be blended with UF } \\
\text { filtrated CSG produced water for irrigation. }\end{array}$ & [69] \\
\hline 5 & $\mathrm{MF}+$ Activated carbon $+\mathrm{MD}$ & Reuse & $\begin{array}{c}\text { TDS removal }>99.6 \% ; \text { TOC removal }>90 \% ; \\
\text { Recovery }=70 \%\end{array}$ & {$[70]$} \\
\hline 6 & $\begin{array}{l}\text { Aeration tank }+ \text { air floatation }+ \text { sand filter }+ \\
\text { UF; Pilot scale. }\end{array}$ & $\begin{array}{l}\text { Discharge } \\
\text { or } \\
\text { reinjection }\end{array}$ & $\begin{array}{l}\text { Feed: Produced water from Daqing oil field. Oil } \\
\text { in product }<0.5 \mathrm{mg} / \mathrm{L} \text {; SS contents }<1.0 \mathrm{mg} / \mathrm{L} \text {; } \\
\text { The effluent can meet discharging or injection } \\
\text { water quality standard. }\end{array}$ & [44] \\
\hline 7 & $\begin{array}{c}\text { Warm Softening }\left(\mathrm{pH} 9.5 ; 71^{\circ} \mathrm{C}\right)+\text { Cooling } \\
\left(40^{\circ} \mathrm{C}\right)+\text { Equalization Storage + Pressure } \\
\text { Filtration + Cartridge Filtration + Reverse } \\
\text { Osmosis }\left(\mathrm{pH} 9.5 ; 40^{\circ} \mathrm{C}\right)+\text { Disinfection }+ \\
\text { Sludge Handling Reject + Disposal; Pilot } \\
\text { scale. }\end{array}$ & $\begin{array}{l}\text { Reuse for } \\
\text { irrigation }\end{array}$ & $\begin{array}{l}\text { Feed: Produced water from Placerita Canyon Oil } \\
\text { Field, Los Angeles County, CA. Warm } \\
\text { softening removed } \sim 95 \% \text { of hardness. RO } \\
\text { removed }>95 \% \text { TDS. About } 90 \% \text { boron removal } \\
\text { was achieved at a } \mathrm{pH} \geq 10.5 \text {. An } 80 \% \text { ammonia } \\
\text { removal was obtained at a } \mathrm{pH} \leq 8.7 \text {. }\end{array}$ & [45] \\
\hline 8 & $\begin{array}{c}\text { Oil/water separator + warm softener + sand } \\
\text { filters + ion exchange softener }+ \text { cartridge } \\
\text { filter }(1.0 \mu \mathrm{m})+\mathrm{RO} \text {; Pilot scale; } \\
\end{array}$ & $\begin{array}{l}\text { Reuse for } \\
\text { irrigation }\end{array}$ & $\begin{array}{l}\text { Remineralization (calcium, magnesium) and } \\
\text { addition some nutrients are needed for irrigation } \\
\text { purpose. }\end{array}$ & [71] \\
\hline 9 & $\begin{array}{l}\text { Cartridge filter }(10 \mu \mathrm{m}+5 \mu \mathrm{m}+1 \mu \mathrm{m})+ \\
\text { Cation exchanger }+ \text { cartridge filter }(0.45 \mu \mathrm{m}) \\
+\mathrm{RO}+\text { constructed wetlands; Pilot scale. }\end{array}$ & $\begin{array}{l}\text { Reuse for } \\
\text { irrigation }\end{array}$ & $\begin{array}{c}\text { Conductivity reduction }=95 \% ; \text { TDS removal }= \\
94 \% ;\end{array}$ & [72] \\
\hline 10 & $\begin{array}{c}\text { Induced gas flotation }+ \text { Nut-shell filter }+ \\
\text { cooking }+ \text { induced gas flotation }+ \text { warm lime } \\
\text { process }+ \text { media filter }+ \text { softener }+ \text { cartridge } \\
\text { filter }+ \text { two-pass } \mathrm{RO}+\text { pH adjustment }+ \\
\text { sodium absorption ratio adjustment }+ \\
\text { constructed wetlands }+ \text { recharge basins; } \\
79,493 \mathrm{~m} / \mathrm{d} \text {; }\end{array}$ & $\begin{array}{l}\text { Reuse for } \\
\text { irrigation }\end{array}$ & $\begin{array}{l}\text { Final effluent: } \text { TDS }=422 \mathrm{mg} / \mathrm{L} ; \text { Alkalinity }=92 \\
\mathrm{mg} / \mathrm{L} ; \mathrm{pH}=7.0 ; \text { Sodium absorption ratio }=17.5 ; \\
\text { TOC }=15.6 \mathrm{mg} / \mathrm{L} ;\end{array}$ & [73] \\
\hline 11 & $\begin{array}{c}\text { Three-phase separator + gravity separator }+ \\
\text { dissolved gas flotation }+ \text { metal removal unit }+ \\
\text { sand filters + VSEP membrane system }+ \\
\text { ammonia absorption column }+ \text { Cartridge filter } \\
(5 \mu \mathrm{m})+\text { RO; Feed flow rate } 90 \mathrm{~m}^{3} / \text { day } \\
\text { (design). }\end{array}$ & $\begin{array}{l}\text { Reuse for } \\
\text { Irrigation }\end{array}$ & $\begin{array}{c}\text { Final effluent: TDS }=160 \mathrm{mg} / \mathrm{L}(\text { TDS in feed }= \\
35,803 \mathrm{mg} / \mathrm{L}) ; \text { Recovery }=45 \% ;\end{array}$ & [74] \\
\hline 12 & $\begin{array}{l}\text { MF + Activated carbon + MD; Estimated for a } \\
100 \mathrm{~m}^{3} / \mathrm{h} \text { designed capacity. }\end{array}$ & $\begin{array}{l}\text { Reuse for } \\
\text { irrigation }\end{array}$ & TDS removal $>99.6 \%$; TOC removal $>90 \%$; & [70] \\
\hline 13 & $\begin{array}{l}\text { Warm Softening }\left(\mathrm{pH} 9.5 ; 71^{\circ} \mathrm{C}\right)+\text { Cooling } \\
\left(40^{\circ} \mathrm{C}\right)+\text { Equalization Storage }+ \text { Pressure } \\
\text { Filtration }+ \text { Cartridge Filtration + Reverse } \\
\text { Osmosis }\left(\mathrm{pH} 10.8 ; 40^{\circ} \mathrm{C}\right)+\mathrm{pH} \text { Adjustment }+ \\
\text { Ion-Exchange for Ammonia }(\mathrm{pH} 7.7 ; \mathrm{T} \\
\text { ambient) + Disinfection + Sludge Handling + } \\
\text { Reject Disposal; Pilot scale. }\end{array}$ & $\begin{array}{l}\text { Reuse for } \\
\text { drinking } \\
\text { water }\end{array}$ & $\begin{array}{l}\text { Feed: Produced water from Placerita Canyon Oil } \\
\text { Field, Los Angeles County, CA. Warm } \\
\text { softening removed } \sim 95 \% \text { of hardness. RO } \\
\text { removed }>95 \% \text { TDS. About } 90 \% \text { boron removal } \\
\text { was achieved at a } \mathrm{pH} \geq 10.5 \text {. An } 80 \% \text { ammonia } \\
\text { removal was obtained at a } \mathrm{pH} \leq 8.7 \text {. }\end{array}$ & [45] \\
\hline 14 & $\begin{array}{l}\text { Warm Softening + Cooling }+\mathrm{MBR}+\mathrm{RO} \\
\text { (two-pass); Conceptual design of } 79,493 \mathrm{~m}^{3} / \mathrm{d} \text {; }\end{array}$ & $\begin{array}{l}\text { Ruse for } \\
\text { potable } \\
\text { water }\end{array}$ & $\begin{aligned} \text { RO product: } & \text { TDS }=1.22 \mathrm{mg} / \mathrm{L} ; \mathrm{pH}=8.0 ; \text { Boron } \\
& =0.12 \mathrm{mg} / \mathrm{L} ; \mathrm{O} \& \mathrm{G}=0.0\end{aligned}$ & [75] \\
\hline 15 & Microfiltration (Zirconia oxide membrane) & Reinjection & TSS and oil in product $<1 \mathrm{mg} / \mathrm{L}$; & [47] \\
\hline
\end{tabular}

\footnotetext{
$\overline{{ }^{*} \text { Corresponding author: igw@,che.itb.ac.id }}$
} 
Another membrane process which is potential for produced water reuse is membrane distillation (MD). Performance of MD in produced water treatment is summarized in Table 4. MD can recover pure water while rejecting the other components. Moreover, the performance of MD is not limited by the osmotic pressure of the solution. Therefore, it is possible to treat a feed water containing a high dissolved solid with a high recovery factor. MD can also be combined with RO membrane [61] to improve the overall water recovery during produced water treatment, especially for reuse purpose. In addition, since MD is a thermallydriven process, it can be directly used to treat a hot produced e.g. from SAGD without cooling [62]. MD can be integrated with a waste heat source to drive the separation process even to reach a concentration of brine solution of $30 \%$ [63]. Tavakkoli et al [64] reported an economic analysis on MD for produced water treatment and showed that by using a waste heat source, the cost of produced water treatment can be significantly reduced from $\$ 5.70 / \mathrm{m}^{3}$ feed to $\$ 0.74 / \mathrm{m}^{3}$ feed.

Among MD configuration, direct contact MD (DCMD), vacuum MD (VMD), sweeping gas MD (SGMD), and air gap MD (AGMD), DCMD is preferred since it does not require external condenser and it requires the simplest equipment and operation $[70,76]$.

Even though membrane shows an excellent performance in treating produced water for reuse purpose, fouling is still the major drawback [28]. This fouling results in a considerable reduction of membrane flux (Fig. 2). Fouling phenomena are inevitable in membrane operation. Strategies for fouling mitigation has been widely studied and can be found in the literature [77-79]. In produced water treatment, several studies used hydrophilic membranes. For example, Xu et al used a hydrophilized PVDF UF membrane for produced water reinjection purpose [82,83]. The membrane exhibited an average $75 \mathrm{LMH}$. The flux recovery was about $95 \%$. The membrane effectively removed oil, turbidity, and TSS to $1 \mathrm{mg} / \mathrm{L}$, $1 \mathrm{NTU}$, and $<2.5 \mathrm{mg} / \mathrm{L}$ (below detection level), respectively, which was able to meet the highest reinjection criteria in China. In addition, the membrane showed an antifouling property. Alzahrani et al [84] investigated the performance of commercial hydrophilic NF and RO membranes for produced water treatment reuse (potable water). The membranes have contact angles of $\sim 23^{\circ}$ and $\sim 37^{\circ}$. It was reported that more hydrophilic and negatively charged membrane shows a less susceptible to fouling [59]. Xu et al [85] investigated UF membrane performance in produced water treatment for reinjection purpose.

Polyethersulfone UF membrane was used in a deadend filtration mode. The membrane showed a high oil removal $(>95 \%)$ with an oil content of lower than 5 $\mathrm{mg} / \mathrm{L}$. They also successfully recovered up to $95 \%$ of membrane flux by combining cleaning solution method of citric acid solution, sodium hydroxide solution, and SDBS solution (membrane is soaked in each solution containing 0.5 wt. $\%$ chemical).

In MD, besides fouling, wetting still becomes the major drawbacks. The treated produced water may still contain impurities which could result in more severe fouling and wetting when fed to MD system. It was reported by Kim et al [86] that the presence oil and grease as well as scaling induced by multivalent ions can enhance the wetting phenomenon. Results of their study suggest a combination of MD and crystallization to reduce scalant content and effectively prevent wetting. Scaling can also be removed by a simple intermittent cleaning operation using diluted acid solution (e.g. $0.32 \mathrm{wt} . \%$ for $10 \mathrm{~min}$ every $24 \mathrm{~h}$ ) which was successfully performed by Zhong et al [87] to maintain permeate flux in 3 cycles operation. Another approach to mitigate membrane wetting is by using a membrane which has an anti-wetting property [88]. For instance, a graphene/PVDF membrane studied by Woo et al [61] showed a stable normalized flux $(85 \%$ final flux after 10 days continuous operation). For a produced water containing dissolved organic matter and surfactant, optimized pretreatment and surface membrane properties are required to minimize flux reduction due to fouling [89].
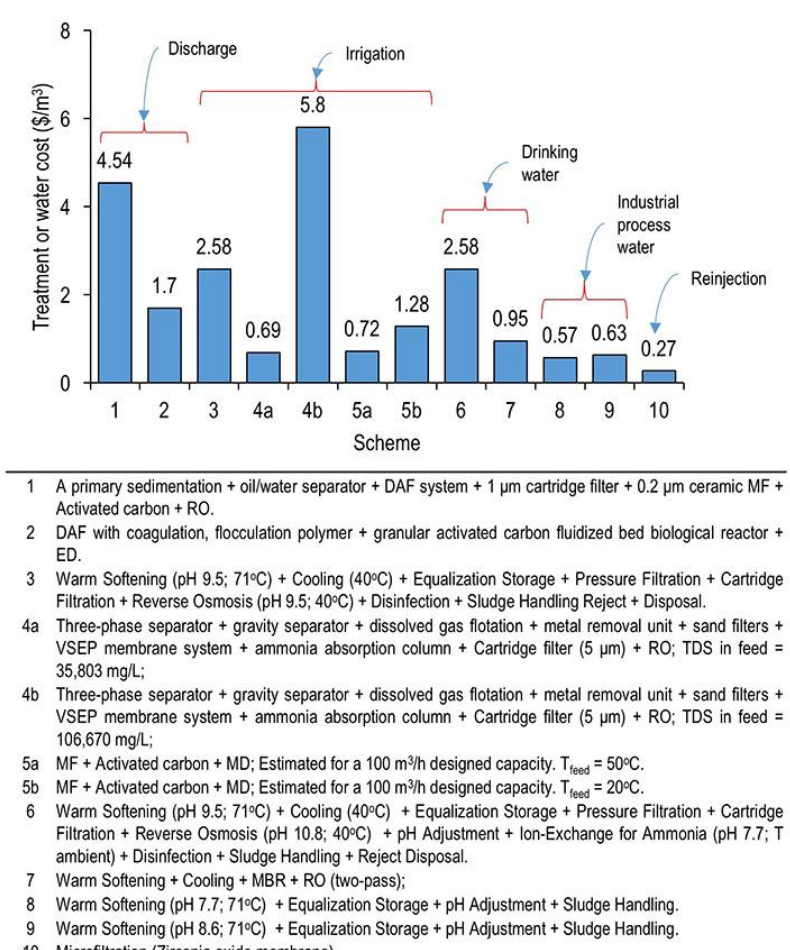
VSEP membrane system + ammonia absorption column + Cartridge filter $(5 \mu \mathrm{m})+\mathrm{RO}$; TDS in feed = $35,803 \mathrm{mg} / \mathrm{L}$;

4b Three-phase separator + gravity separator + dissolved gas flotation + metal removal unit + sand filters + VSEP membrane system + ammonia absorption column + Cartridge filter $(5 \mu \mathrm{m})+\mathrm{RO}$; TDS in feed $=$ $106,670 \mathrm{mg} / \mathrm{L}$;

5a $M F+$ Activated carbon $+M D$; Estimated for a $100 \mathrm{~m}^{3} / \mathrm{h}$ designed capacity. $T_{\text {feed }}=50^{\circ} \mathrm{C}$.

$5 b \mathrm{MF}+$ Activated carbon $+\mathrm{MD} ;$; Estimated for a $100 \mathrm{~m}^{3} / \mathrm{h}$ designed capacity. $T_{\text {feed }}=20^{\circ} \mathrm{C}$.

6 Warm Softening $\left(\mathrm{pH} 9.5 ; 71{ }^{\circ} \mathrm{C}\right)+$ Cooling $\left(40^{\circ} \mathrm{C}\right)+$ Equalization Storage + Pressure Filtration + Cartridge Filtration + Reverse Osmosis $\left(\mathrm{pH} 10.8 ; 40^{\circ} \mathrm{C}\right)+\mathrm{pH}$ Adjustment + Ion-Exchange for Ammonia (pH 7.7; T ambient) + Disinfection + Sludge Handling + Reject Disposal.

Warm Softening + Cooling $+M B R+R O$ (two-pass);

Warm Softening $\left(\mathrm{pH} 7.7 ; 71^{\circ} \mathrm{C}\right)+$ Equalization Storage + $\mathrm{pH}$ Adjustment + Sludge Handling.

Warm Softening $\left(\mathrm{pH} 8.6 ; 71^{\circ} \mathrm{C}\right)+$ Equalization Storage + pH Adjustment + Sludge Handling.

10 Microfiltration (Zirconia oxide membrane)

Fig. 3. Total cost of schemes used in produced water treatment (data from refs. $[42,45,47,70,74,75,95]$ )

\footnotetext{
$\overline{{ }^{*} \text { Corresponding author: igw@che.itb.ac.id }}$
} 
Table 4. MD for produced water treatment

\begin{tabular}{|c|c|c|c|c|}
\hline Process & Membrane & Operational conditions & Results & Ref. \\
\hline MD & PTFE $(0.03 \mu \mathrm{m})$ & $\begin{array}{l}\text { DCMD; Tf }=128^{\circ} \mathrm{C} ; \mathrm{Tc}=20^{\circ} \mathrm{C} ; 2-3 \text { atm; Feed }= \\
\text { Synthetic PW }(\mathrm{NaCl}=3000 ; \text { Phenol }=45 ; \text { Cresol }= \\
\text { 45; Naphtenic acid = 10; all in mg } / \mathrm{L}) ;\end{array}$ & Flux $=195 \mathrm{LMH}$ & [62] \\
\hline MD & PTFE $(0.2 \mu \mathrm{m})$ & $\begin{array}{l}\text { AGMP; Tf }=40-80{ }^{\circ} \mathrm{C} ; \mathrm{Tc}=5-25{ }^{\circ} \mathrm{C} ; \mathrm{Feed}= \\
\text { Produced water from ARAMCO (TDS }=187,440 \\
\mathrm{mg} / \mathrm{L}) ;\end{array}$ & $\begin{array}{l}\text { Flux }=7-18 \quad \text { LMH; Salt } \\
\text { rejection }>99 \% ; \quad \text { Organic } \\
\text { rejection }>98 \% ;\end{array}$ & [90] \\
\hline MD & PP & $\begin{array}{l}\mathrm{DCMD} ; \mathrm{Tf}=50-85{ }^{\circ} \mathrm{C} ; \mathrm{Tc}=20{ }^{\circ} \mathrm{C} ; \mathrm{Feed}= \\
\text { Produced water from Chevron }(\mathrm{TDS}=7622(\mathrm{~S} 1) \\
12,040(\mathrm{~S} 2) ; 35,000(\mathrm{~S} 3)) ;\end{array}$ & $\begin{array}{l}\text { Flux }=15-20 \text { LMH }\left(\mathrm{T}_{\mathrm{f}}=\right. \\
\left.80^{\circ} \mathrm{C}\right) ; \text { Recovery }=80 \% ; \text { Salt } \\
\text { rejection }>99 \% ;\end{array}$ & [91] \\
\hline MD & $\mathrm{PP}(0.2 \mu \mathrm{m})$ & $\begin{array}{l}\mathrm{DCMD} ; \mathrm{Tf}=50-70{ }^{\circ} \mathrm{C} ; \mathrm{Tc}=25^{\circ} \mathrm{C} ; \mathrm{Feed}=\mathrm{PW} \\
\text { treated by MF and } \mathrm{AC}(\mathrm{TDS}=247,900 ; \mathrm{TC}=40.7 ; \\
\text { all in } \mathrm{mg} / \mathrm{L}) ;\end{array}$ & $\begin{array}{l}\text { Flux = 6-14 LMH; } \mathrm{R}_{\mathrm{TDS}}= \\
\text { 99.7\%; } \mathrm{R}_{\mathrm{TC}}=92.9 \% ;\end{array}$ & [70] \\
\hline MD & $\operatorname{PVDF}(0.29 \mu \mathrm{m})$ & $\begin{array}{l}\text { DCMD; Tf }=50-70{ }^{\circ} \mathrm{C} ; \mathrm{Tc}=25^{\circ} \mathrm{C} ; \mathrm{Feed}=\mathrm{PW} \\
\text { treated by MF and AC }(\mathrm{TDS}=247,900 ; \mathrm{TC}=40.7 ; \\
\text { all in } \mathrm{mg} / \mathrm{L}) ;\end{array}$ & $\begin{array}{l}\text { Flux }=4-11 \text { LMH; RTDS }= \\
99.8 \% ; \mathrm{R}_{\mathrm{TC}}=95.0 \% ;\end{array}$ & [70] \\
\hline MD & $\operatorname{PVDF}(0.43 \mu \mathrm{m})$ & $\begin{array}{l}\mathrm{DCMD} ; \mathrm{Tf}=50-70{ }^{\circ} \mathrm{C} ; \mathrm{Tc}=25^{\circ} \mathrm{C} ; \mathrm{Feed}=\mathrm{PW} \\
\text { treated by MF and } \mathrm{AC}(\mathrm{TDS}=247,900 ; \mathrm{TC}=40.7 ; \\
\text { all in } \mathrm{mg} / \mathrm{L}) ;\end{array}$ & $\begin{array}{l}\text { Flux }=3-7 \text { LMH; R } \text { RDS }^{=} \\
99.8 \% ; \mathrm{R}_{\mathrm{TC}}=90.6 \% ;\end{array}$ & [70] \\
\hline MD & $\begin{array}{l}\text { MWCNT/PP }(0.846 \\
\mu \mathrm{m})\end{array}$ & $\begin{array}{l}\text { VMD; Tf }=55{ }^{\circ} \mathrm{C} ; \mathrm{Tc}=15^{\circ} \mathrm{C} ; \text { Feed }=\text { Oilfield } \\
\text { produced wastewater }(\mathrm{TDS}=230,000 \mathrm{mg} / \mathrm{L})\end{array}$ & $\begin{array}{l}\text { Flux }=19.6 \text { LMH; RTDS }= \\
99.9 \% ;\end{array}$ & [92] \\
\hline MD & $\begin{array}{l}\mathrm{SiNP} / \mathrm{PVDF} \quad(0.45 \\
\mu \mathrm{m})\end{array}$ & $\begin{array}{l}\text { DCMD; } \mathrm{Tf}=60^{\circ} \mathrm{C} ; \mathrm{Tc}=20^{\circ} \mathrm{C} ; \mathrm{Feed}=\text { prefiltered } \\
\text { shale produced wastewater }(\mathrm{TDS}=101,000 ; \mathrm{O} \& \mathrm{G} \\
=2 ; \text { Hardness }=5910 \text { all in } \mathrm{mg} / \mathrm{L}) ;\end{array}$ & Flux = 13.6 LMH; & [93] \\
\hline MD & $\operatorname{PVDF}(0.22 \mu \mathrm{m})$ & $\begin{array}{l}\text { DCMD; } \mathrm{Tf}=60-80^{\circ} \mathrm{C} ; \mathrm{Tc}=20^{\circ} \mathrm{C} ; \mathrm{Feed}= \\
\text { synthetic } \mathrm{NaCl} / \mathrm{NaHCO} 3 \text { solution }(1 \mathrm{mM} \mathrm{NaCl} / 1 \\
\left.\mathrm{mM} \mathrm{NaHCO}_{3} \text { and } 500 \mathrm{mM} \mathrm{NaCl} / 25 \mathrm{mM} \mathrm{NaHCO} 3\right)\end{array}$ & $\begin{array}{l}\text { Flux }=18.8-41.9 \mathrm{LMH} ; \mathrm{R}= \\
>99.5 \% \text { and }>99.8 \%\end{array}$ & [94] \\
\hline
\end{tabular}

Moreover, membrane technology also faces other challenges including a high permeability and product, easy to clean, and chemical and thermal stability [32]. Chemical stability is needed since produced water contains hydrocarbon which may result in membrane damage, especially for a polymeric membrane. Ceramic membrane is used in produced water treatment due to its high chemical stability. This is beneficial in either filtration and cleaning process since a polymeric membrane is generally sensitive to hydrocarbon in produced water as well as chemical used in cleaning. The higher chemical stability will lead to a longer membrane lifetime. Ceramic membrane may also undergo a harsh chemical cleaning resulting in a better membrane regeneration. Therefore, the ceramic membrane can be potential material in produced water treatment.

Weschenfelder et al [25] used zirconia multichannel UF membrane for removal of suspended solids and oil. The membrane can remove suspended solids completely with an oil content of $<5 \mathrm{mg} / \mathrm{L}$. This shows the potential applications of ceramic UF membrane in produced water treatment for reinjection. $\mathrm{Li}$ and Lee reported that ceramic MF membrane can remove oil and suspended solids from produced water effectively [31]. Ebrahimi et al used ceramic hollow fiber membrane $\left(\mathrm{Al}_{2} \mathrm{O}_{3} ; d_{\text {in }}=2\right.$ $\mathrm{mm}, d_{\text {out }}=4 \mathrm{~mm}$ ) with pore size diameter of $40 \mathrm{~nm}$ for produced water treatment [32]. By using this membrane, $>99.5 \%$ of oil and up to $94 \%$ of TOC can be removed from produced water dewatering tank samples. The performance of the membrane is better than membrane used in their previous study [66] which only reached $49 \%$ TOC removal with comparable oil removal. MFItype zeolite membrane can be used to remove dissolved solid replacing the polymeric RO membrane [96]. MFI zeolite membrane gives more than $99 \%$ ion rejection with water flux around $101 . \mathrm{m}^{-2} \cdot \mathrm{h}^{-1}$ [31]. This type of membrane is also able to remove dissolved organic content [97]. However, the cost of the inorganic membrane is higher than the polymeric membrane. Therefore, producing a low-cost inorganic membrane is needed for commercial scale plant.

Fig. 3 shows the total cost of different schemes and purposes used in produced water treatment including discharge and reuse (for industrial purposes, drinking water, irrigation, and reinjection). It is obvious that the scheme and the final purpose of the treatment determine the treatment cost. The treatment cost increases along with the increasing quality of the end product. This is due to the more complex system used. In addition, additional nutrient may be required, for example, for irrigation purpose. Moreover, produced water characteristic also determine the overall treatment cost. This effect is showed by scheme $4 \mathrm{a}$ and $\mathrm{b}$ and $5 \mathrm{a}$ and $\mathrm{b}$ (Fig. 3). A higher TDS content results in an increased treatment cost involving RO. Meanwhile, a higher produced water temperature fed to a system involving MD may reduce the treatment cost. Among the treatment purposes, reinjection may be an interesting option. Since the produced water characteristic is almost similar to the formation water, a simpler treatment system may be used to achieve an acceptable water injection quality rather than the other reuse purposes. Therefore, the treatment cost is lower. Moreover, reinjection is also beneficial for both economic and environmental aspects. 


\section{Future challenges}

Produced water has become a global environmental issue due to its huge volume and toxicity that may pose detrimental effects on receiving environment. Several approaches have been proposed to provide a strategy for produced water handling such as reinjection, reuse, or discharge. Among the reuse purposes, reinjection is the most attractive option. This is due to the attractive goal of both saving fresh water usage along with near-zero discharge. It is also viewed as a more cost-effective alternative rather than discharge and other reuse purposes. With various advantages, membrane technology has been increasingly used in produced water treatment replacing the conventional technologies. However, fouling is a major drawback of membrane processes in this application which needs to be controlled. Various strategies have been conducted to solve this problem such as choosing an appropriate cleaning procedure and using a membrane with an antifouling property. For MD, an anti-wetting membrane is also needed to maintain a stable flux for a long-term operation. Since produced water contains hydrocarbon, a chemically stable membrane such as inorganic membrane was recommended by several studies to avoid membrane degradation. This stability is also important to obtain a better membrane regeneration by a harsh chemical cleaning. However, a lower cost inorganic membrane may be needed.

\section{References}

1. A. Fakhru'l-Razi, A. Pendashteh, L.C. Abdullah, D.R.A. Biak, S.S. Madaeni, Z.Z. Abidin, J. Hazard. Mater., 170, 530 (2009).

2. B. Gargouri, O.D. Gargouri, B. Gargouri, S.K. Trabelsi, R. Abdelhedi, M. Bouaziz, Chemosphere, 117, 309 (2014).

3. J.A. Veil, M.G. Puder, D. Elcock, R.J. Redweik Jr, A white paper describing produced water from production of crude oil, natural gas, and coal bed methane., Argonne National Lab., IL (US), (2004).

4. C.E. Clark, J.A. Veil, Produced water volumes and management practices in the United States., Argonne National Laboratory (ANL), (2009).

5. G.T. Tellez, N. Nirmalakhandan, J.L. GardeaTorresdey, Adv. Environ. Res., 6, 455 (2002).

6. J.D. Arthur, B.G. Langhus, C. Patel, All Consult. LLC, Tulsa, OK, (2005).

7. N.F. Himma, S. Anisah, N. Prasetya, I.G. Wenten, J. Polym. Eng., 36, 329 (2016).

8. Khoiruddin, I.N. Widiasa, I.G. Wenten, J. Food Eng., 133, 40 (2014).

9. M. Purwasasmita, D. Kurnia, F.C. Mandias, Khoiruddin, I.G. Wenten, Food Bioprod. Process., 94, 180 (2015).

10. I.G. Wenten, P.T. Dharmawijaya, P.T.P. Aryanti, R.R. Mukti, Khoiruddin, RSC Adv., 7, 29520 (2017).

11. I.G. Wenten, Khoiruddin, J. Eng. Sci. Technol., 11, 916 (2016).
12. I.G. Wenten, I.N. Widiasa, Desalination, 149, 279 (2002).

13. I.G. Wenten, H. Julian, N.T. Panjaitan, Desalination, 306, 29 (2012).

14. D. Ariono, M. Purwasasmita, I.G. Wenten, J. Eng. Technol. Sci., 48, 367 (2016).

15. P.T.P. Aryanti, M. Sianipar, M. Zunita, I.G. Wenten, Membr. Water Treat., 8, 463 (2017).

16. Khoiruddin, D. Ariono, Subagjo, I.G. Wenten, J. Appl. Polym. Sci., (2017).

17. I.G. Wenten, Khoiruddin, Desalination, 391, 112 (2016).

18. I.G. Wenten, Khoiruddin, F. Arfianto, Zudiharto, Desalination, 314, 109 (2013).

19. A.K. Wardani, A.N. Hakim, Khoiruddin, I.G. Wenten, Water Sci. Technol., 75, 2891 (2017).

20. S. Munirasu, M.A. Haija, F. Banat, Process Saf. Environ. Prot., 100, 183 (2016).

21. S. Alzahrani, A.W. Mohammad, J. Water Process Eng., 4, 107 (2014).

22. J.M. Dickhout, J. Moreno, P.M. Biesheuvel, L. Boels, R.G.H. Lammertink, W.M. de Vos, J. Colloid Interface Sci., 487, 523 (2017).

23. A.R.P. da Motta, C.P. Borges, A. Kiperstok, K.P. Esquerre, P.M. Araujo, L.P.N. Branco, Eng. Sanit. E Ambient., 18, 15 (2013).

24. S. Mondal, Rev. Chem. Eng., 32, 611 (2016).

25. S.E. Weschenfelder, A.M.T. Louvisse, C.P. Borges, E. Meabe, J. Izquierdo, J.C. Campos, J. Pet. Sci. Eng., 131, 51 (2015).

26. D. Robinson, Filtr. Sep., 47, 14 (2010).

27. Society of Petroleum Engineers, Challenges in Reusing Produced Water, http://www.spe.org/industry/challenges-in-reusingproduced-water.php .

28. M. Ebrahimi, K.S. Ashaghi, L. Engel, D. Willershausen, P. Mund, P. Bolduan, P. Czermak, Desalination, 245, 533 (2009).

29. E.T. Igunnu, G.Z. Chen, Int. J. Low-Carbon Technol., 9, 157 (2012).

30. J.E. Horner, J.W. Castle, J.H. Rodgers, Ecotoxicol. Environ. Saf., 74, 989 (2011).

31. L. Li, R. Lee, Sep. Sci. Technol., 44, 3455 (2009).

32. M. Ebrahimi, S. Kerker, S. Daume, M. Geile, F. Ehlen, I. Unger, S. Schütz, P. Czermak, Desalin. Water Treat., 55, 3554 (2015).

33. D. Robinson, Filtr. + Sep., 50, 20 (2013).

34. R.C. Evans, Developments in Environmental Protection Related to Produced Water Treatments and Disposal (Produced Water Re-Injection), in: SPE Heal. Saf. Environ. Oil Gas Explor. Prod. Conf., Society of Petroleum Engineers, Jakarta (1994).

35. D. Robinson, Filtr. + Sep., 50, 40 (2013).

36. A. Hussain, J. Minier-Matar, A. Janson, S. Gharfeh, S. Adham, Advanced technologies for produced water treatment and reuse, in: Soc. Pet. Eng. - Int. Pet. Technol. Conf. 2014, IPTC 2014 Unlocking Energy Through Innov. Technol. Capab., ConocoPhillips, United States (2014), pp. 16211631.

37. E.J. Sullivan Graham, A.C. Jakle, F.D. Martin, 
Water Int., 40, 809 (2015).

38. R.R. Nair, E. Protasova, T. Bilstad, S. Strand, Reuse of produced water by membranes for enhanced oil recovery, in: Proc. - SPE Annu. Tech. Conf. Exhib., Department of Mathematics and Natural Science, United States (2016).

39. J. Ochi, D. Dexheimer, P. V Corpel, SPE Prod. Oper., 29, 192 (2014).

40. R.W. Paige, L.R. Murray, Re-injection of produced water - Field experience and current understanding, in: Rock Mech. Pet. Eng. 29-31 August, Delft, Netherlands, Society of Petroleum Engineers, (1994), pp. 731-738.

41. D. Robinson, Filtr. Sep., 50, 36 (2013).

42. M. Çakmakce, N. Kayaalp, I. Koyuncu, Desalination, 222, 176 (2008).

43. J.C. Campos, R.M.H. Borges, A.M. Oliveira Filho, R. Nobrega, G.L. Sant'Anna, Water Res., 36, 95 (2002).

44. X. Qiao, Z. Zhang, J. Yu, X. Ye, Desalination, 225, 113 (2008).

45. R. Funston, R. Ganesh, L.Y.C. Leong, Gr. Water Prot. Counc. (GWPC), Color. Spring, US, 16 (2002).

46. C.H. Webb, L. Nagghappan, G. Smart, J. Hoblitzell, R. Franks, Desalination of OilfieldProduced Water at the San Ardo Water Reclamation Facility, CA, in: SPE West. Reg. Meet. 2009, Society of Petroleum Engineers, San Jose, California (2009), pp. 565-585.

47. S.E. Weschenfelder, C.P. Borges, J.C. Campos, J. Memb. Sci., 495, 242 (2015).

48. X.-H. Zhen, S.-L. Yu, B.-H. Wang, H.-F. Zheng, J. Environ. Sci. (China), 18, 1077 (2006).

49. G.F. Doran, F.H. Carini, D.A. Fruth, J.A. Drago, L.Y.C. Leong, Evaluation of technologies to treat oil field produced water to drinking water or reuse quality, in: Proc. - SPE Annu. West. Reg. Meet., ARCO Western Energy (1997), pp. 811-822.

50. M. Gamal Khedr, Desalin. Water Treat., 55, 3460 (2015)

51. Q. Helmy, E. Kardena, J. Pet. Environ. Biotechnol., 6, 1 (2015).

52. J. Kus, R. Card, J. Can. Pet. Technol., 23, 66 (1984).

53. J. Cao, S. Monroe, H. Sun, L. Li, Cost-efficient and improved treatment technology for produced water reuse, in: Proc. - SPE Annu. Tech. Conf. Exhib., Baker Hughes Incorporated, United States (2015), pp. $974-982$.

54. F. Hum, P. Tsang, A. Kantzas, T. Harding, Is it possible to treat produced water for recycle and beneficial reuse?, in: SPE/PS-CIM/CHOA Int. Therm. Oper. Heavy Oil Symp. Proc., TIPM Laboratory, Canada (2005), pp. A135-A148.

55. J. Mueller, Y. Cen, R.H. Davis, J. Memb. Sci., 129, 221 (1997).

56. C. Yang, G. Zhang, N. Xu, J. Shi, J. Memb. Sci., 142, 235 (1998).

57. J. Zhong, X. Sun, C. Wang, Sep. Purif. Technol., 32, 93 (2003).

58. A. Salahi, M. Abbasi, T. Mohammadi,
Desalination, 251, 153 (2010).

59. M. Hayatbakhsh, M. Sadrzadeh, D. Pernitsky, S. Bhattacharjee, J. Hajinasiri, Desalin. Water Treat., 57, 14869 (2016).

60. Y. Sun, W.-X. Shi, J. Xu, M. Yu, L.-Z. Jia, $A d v$. Mater. Res., 113-116, 1541 (2010).

61. Y.C. Woo, Y. Kim, W.-G. Shim, L.D. Tijing, M. Yao, L.D. Nghiem, J.-S. Choi, S.-H. Kim, H.K. Shon, J. Memb. Sci., 513, 74 (2016).

62. D. Singh, K.K. Sirkar, J. Memb. Sci., 389, 380 (2012).

63. O.R. Lokare, S. Tavakkoli, G. Rodriguez, V. Khanna, R.D. Vidic, Desalination, 413, 144 (2017).

64. S. Tavakkoli, O.R. Lokare, R.D. Vidic, V. Khanna, Desalination, 416, 24 (2017).

65. O.A. Al-Harbi, M. Mujtaba Khan, C. Özgür, $J$. Aust. Ceram. Soc., 53, 883 (2017).

66. M. Ebrahimi, D. Willershausen, K.S. Ashaghi, L. Engel, L. Placido, P. Mund, P. Bolduan, P. Czermak, Desalination, 250, 991 (2010).

67. S.J. Maguire-Boyle, J.E. Huseman, T.J. Ainscough, D.L. Oatley-Radcliffe, A.A. Alabdulkarem, S.F. Al-Mojil, A.R. Barron, Sci. Rep., 7, (2017).

68. M. Ebrahimi, Z. Kovacs, M. Schneider, P. Mund, P. Bolduan, P. Czermak, Desalin. Water Treat., 42, 17 (2012).

69. L.D. Nghiem, C. Elters, A. Simon, T. Tatsuya, W. Price, Sep. Purif. Technol., 146, 94 (2015).

70. F. Macedonio, A. Ali, T. Poerio, E. El-Sayed, E. Drioli, M. Abdel-Jawad, Sep. Purif. Technol., 126, 69 (2014).

71. M. Melo, H. Schluter, J. Ferreira, R. Magda, A. Júnior, O. de Aquino, Desalination, 250, 1016 (2010).

72. C. Murray-Gulde, J.E. Heatley, T. Karanfil, J.H.R. Jr., J.E. Myers, Water Res., 37, 705 (2003).

73. J.E. Myers, Chevron San Ardo Facility Unit (SAFU) beneficial produced water reuse for irrigation, in: Soc. Pet. Eng. - SPE Int. Conf. Heal. Saf. Environ. 2014 Journey Contin., PE, Chevron, United States (2014), pp. 687-697.

74. V. Piemonte, M. Prisciandaro, L. Mascis, L. Di Paola, D. Barba, Desalin. Water Treat., 55, 565 (2015).

75. P.B. Tsang, C.J. Martin, Economic Evaluation of Treating Oilfield Produced Water for Potable Use, in: SPE Int. Therm. Oper. Heavy Oil Symp. West. Reg. Meet., Society of Petroleum Engineers, (2004), p. SPE 86984.

76. E. Drioli, A. Criscuoli, E. Curcio, Membrane contactors: fundamentals, applications and potentialities, Elsevier, (2011).

77. H. Lin, W. Peng, M. Zhang, J. Chen, H. Hong, Y. Zhang, Desalination, 314, 169 (2013).

78. N. Hilal, O.O. Ogunbiyi, N.J. Miles, R. Nigmatullin, Sep. Sci. Technol., 40, 1957 (2005).

79. M.F.A. Goosen, S.S. Sablani, H. Al-Hinai, S. Al-Obeidani, R. Al-Belushi, D. Jackson, Sep. Sci. Technol., 39, 2261 (2005).

80. T.D. Kusworo, N. Aryanti, M.M.H. Firdaus, H. Sukmawati, Surface modification of cellulose 
acetate membrane using thermal annealing to enhance produced water treatment, in: AIP Conf. Proc., (2015).

81. X. Yang, R. Wang, A.G. Fane, C.Y. Tang, I.G. Wenten, Desalin. Water Treat., 51, 3604 (2013).

82. J. Xu, W.-X. Shi, S.-L. Yu, W.-M. Qu, Adv. Mater. Res., 113-116, 1266 (2010).

83. J. Xu, C. Ma, B. Cao, J. Bao, Y. Sun, W. Shi, S. Yu, Process Saf. Environ. Prot., 104, 564 (2016).

84. S. Alzahrani, A.W. Mohammad, P. Abdullah, O. Jaafar, J. Environ. Chem. Eng., 1, 1341 (2013).

85. J. Xu, C. Ma, Y. Sun, W. Shi, S. Yu, L. Wang, Desalin. Water Treat., 61, 42 (2017).

86. J. Kim, H. Kwon, S. Lee, S. Lee, S. Hong, Desalination, 403, 172 (2017).

87. W. Zhong, H. Li, Y. Ye, V. Chen, Desalination, 393, 52 (2016).

88. N.G.P. Chew, S. Zhao, C.H. Loh, N. Permogorov, R. Wang, J. Memb. Sci., 528, 126 (2017).

89. M. Malmali, P. Fyfe, D. Lincicome, K. Sardari, S.R. Wickramasinghe, Sep. Sci. Technol., 52, 266 (2017).

90. A. Alkhudhiri, N. Darwish, N. Hilal, Desalination, 309, 46 (2013).

91. D. Singh, P. Prakash, K.K. Sirkar, Ind. Eng. Chem. Res., 52, 13439 (2013).

92. K. Okiel, A.H.M. El-Aassar, T. Temraz, S. ElEtriby, H.A. Shawky, Desalin. Water Treat., 57, 10995 (2016).

93. C. Boo, J. Lee, M. Elimelech, Environ. Sci. Technol., 50, 12275 (2016).

94. D.-W. Cho, H. Song, K. Yoon, S. Kim, J. Han, J. Cho, Water (Switzerland), 8, (2016).

95. J.A. Miller, A.W. Lawrence, R.F. Hickey, T.D. Hayes, (n.d.).

96. N. Liu, J. Lu, A. Li, R. Lee, Factors determining the reverse osmosis performance of zeolite membranes on produced water purification, in: Proc. - SPE Int. Symp. Oilf. Chem., (2007), pp. 399-405.

97. N. Liu, L. Li, B. McPherson, R. Lee, J. Memb. Sci, 325, 357 (2008). 